\title{
Is primary meningococcal arthritis in children more frequent than we expect? Two pediatric case reports revealed by molecular test
}

\author{
S. Ricci ${ }^{1 *}$ (D, A. Montemaggi ${ }^{2}$, F. Nieddu ${ }^{2}$, D. Serranti ${ }^{2}$, G. Indolfi $^{3}$, M. Moriondo $^{1}$ and C. Azzari ${ }^{1}$
}

\begin{abstract}
Background: Primary meningococcal arthritis is a rare infectious disease that occurs in less than 3\% of meningococcal infections and is characterized by arthritis without meningitis, fever, rash, or hemodynamic instability Barahona [Case Rep Orthop 4696014:2017 ]. There are no validated clinical criteria that can be used for the diagnosis. We present two pediatric cases of atypical presentation of meningococcal disease revealed by molecular tests.

Case presentation: The clinical presentation of the two children (6- and 9-years-old) was characterized by signs of arthritis. By Real Time Polymerase Chain Reaction (RT-PCR), we identified N. meningitidis serogroup $Y$ in the joint fluid in both cases. After specific antimicrobial treatment, the clinical conditions of the two patients quickly improved during hospitalization. Conclusions. We believe that the incidence of meningococcal arthritis could be underestimated in those settings where the use of RT-PCR is limited. Clearer data on the incidence of meningococcal disease would help to design specific treatments and the best possible national vaccine strategies [Fiji Sci Rep 23:39784, 2016, J Infect 67:385-90, 2013].
\end{abstract}

Keywords: Primary meningococcal arthritis (PMA), N. meningitidis, Invasive meningococcal disease (IMD), RT-PCR

\section{Background}

Primary meningococcal arthritis (PMA) is an uncommon form of meningococcal disease presenting as isolated septic arthritis without any other signs of invasive meningococcal disease (IMD). It is clinically impossible to differentiate PMA from other types of septic arthritis. Synovial fluid culture and molecular tests are pivotal the confirmation of PMA [1-7]. We discuss two PMA cases diagnosed by Real Time Polymerase Chain Reaction (RT-PCR) following the admission in our hospital of two children in January and March 2017, respectively.

\footnotetext{
* Correspondence: slv.ricci@gmail.com

${ }^{1}$ Immunology Division, Section of Pediatrics, Department of Health Sciences, University of Florence and Meyer Children's University Hospital, Florence, Italy Full list of author information is available at the end of the article
}

\section{Cases presentation}

Case 1

A 6-year-old female with Down syndrome presented at our emergency department with a 24-h history of fever, left hip joint pain and limping. She had been previously diagnosed with compensated mitral valve prolapse, reported a past episode of pneumonia and underwent adenotonsillectomy for obstructive apnea 3 years earlier. One month before admission she traveled to Cuba with her family. On admission, she was febrile with left coxofemoral joint pain and movement impairment. Blood tests showed increased white cells count (WCC) with neutrophilia (WCC 23070/ $\mathrm{mm}^{3}, \mathrm{~N} 90 \%$ ) and a raised C reactive protein (CRP $10 \mathrm{mg} /$ $\mathrm{dl}$, normal $<0.29 \mathrm{mg} / \mathrm{dl}$ ). A left coxofemoral ultrasound documented a $10 \mathrm{~mm}$ intra-articular fluid effusion.

\section{Case 2}

A 9-year-old male attended the emergency department with a 3-days history of right ankle joint pain 
non-responding to non-steroidal anti-inflammatory drugs, limited joint movement and apyrexial. The child had been adopted from Hungary the year before and was diagnosed and treated for Toxocariasis at the baseline health screening as an adopted child. There was no recent history of close contact with other children and no history of respiratory or urinary tract infections.

Blood tests showed increased WCC with neutrophilia (WCC 13700/mm3, 70\% N), C reactive protein (CRP 11 $\mathrm{mg} / \mathrm{dl}$, normal $<0.29 \mathrm{mg} / \mathrm{dl}$ ), and erythrocyte sedimentation rate $($ ESR $64 \mathrm{~mm} / \mathrm{h}$, normal $<15 \mathrm{~mm} / \mathrm{h}$ ). A right hip $\mathrm{X}$-ray was normal, whereas right hip ultrasound showed an 11-mm intra-articular effusion and swelling of the right synovial capsule. He did not present any other location of arthritis or arthralgia.

\section{Case 1 and case 2: Diagnosis, treatment, and outcome}

Both patients were admitted to our pediatric ward to be started on empirical antimicrobial treatment with intravenous ceftriaxone and oxacillin for suspected septic arthritis, as recommended in the literature [8]. RT-PCR was performed on the synovial fluid on day 3 of antibiotic therapy: $c t r A$ gene and specific primers and probes for A (sacB gene) and B, C, W,Y serogroups (siaD B, siaD $\mathrm{C}$, siaD W and Y, respectively) were used for the detection of N.meningitidis as we have previously described [9]. The test resulted positive for $N$. meningitidis serogroup Y (NmY). Multilocus sequence typing (MLST) on synovial fluid revealed the presence of the finetype $\mathrm{Y}$ P1.5-2, 10-2: F2-13: ST-23 (cc23) in both samples. According to local IMD treatment guidelines, antibiotic therapy was switched to penicillin $G$ and intravenous ceftriaxone was continued while waiting for culture confirmation and MIC (minimal inhibitory concentration) value for penicillin; oxacillin was stopped and prophylaxis for contacts was started [8]. RT-PCR test for $N$. meningitidis in blood and hemoculture (after $24 \mathrm{~h}$ of antimicrobial therapy with ceftriaxone and oxacillin) and a synovial fluid culture test (after $48 \mathrm{~h}$ of antimicrobial therapy) were negative in both cases. No bone involvement was detected with a coxofemoral joint MRI performed in either case (Fig. 1). Our patients' clinical conditions quickly improved during hospitalization. Intravenous antimicrobial therapy was continued for 15 days and was subsequently switched to oral treatment for a total of 6 weeks. Both patients were asymptomatic at a follow-up arranged 4 months after discharge.

Immunological laboratory screening including serum immunoglobulin, IgG subclasses, antibody response, immunophenotyping, DHR test and complement function assay ( $\mathrm{CH} 50)$ was performed to investigate any predisposing immunologic deficiency. Down syndrome could be associated with humoral and cellular defects. In case 1, lymphocyte subpopulation analysis showed a mildly reduced CD4+ lymphocytes (24\%), with a normal absolute value for age (av: 416 cells $/ \mu \mathrm{L}$ ). The immunological screening was totally normal in the second case. Neither patient had been previously vaccinated with the quadrivalent meningococcal vaccine which was subsequently offered at follow-up (quadrivalent meningococcal conjugate vaccine and $4 \mathrm{CMen} B$ vaccine).

\section{Discussion and conclusions}

$N$. meningitidis $(\mathrm{Nm})$ is a well-known pathogen in meningitis and sepsis, but other end-organ manifestations of IMD such as pneumonia and septic arthritis are also described. Meningococcal epidemiology is unpredictable and not completely understood leading to a high variability in the incidence of IMD [10]. RT-PCR is approximately 3-times more sensitive than culture in identifying $N$. meningitidis from biological samples, therefore meningococcal infections might be underdiagnosed when using culture-based methods alone, especially in the event of an unusual presentation such as arthritis [9, 11-15]. Meningococcal arthritis is a recognized complication of meningococcal infections reported in up to $7.5 \%$ of cases in association with IMD. Nevertheless, PMA is an unusual presentation reported in $2.6 \%$ of meningococcal infections and has an excellent prognosis $[1,16]$. According to Barahona et al. [1], PMA is considered a rare entity, accounting for $1.5-1.8 \%$ of all pediatric cases of pyogenic arthritis
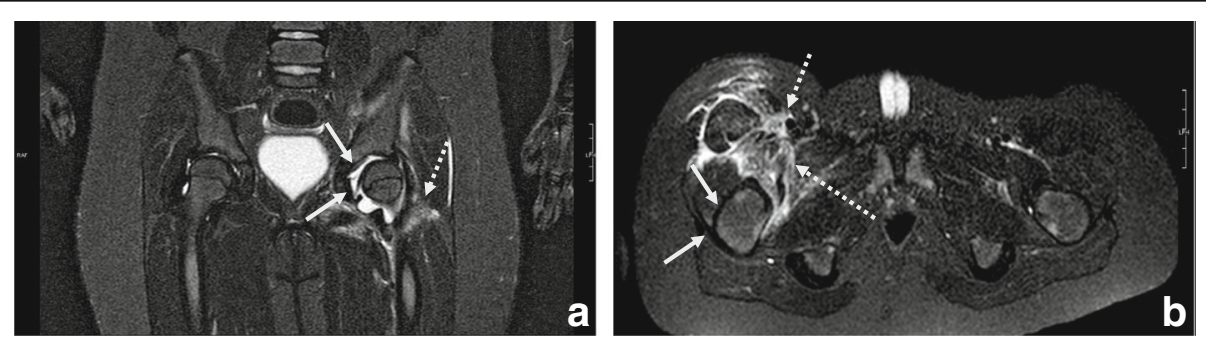

Fig. 1 Most significant coxofemoral joint MRI findings performed on the third day after admission in both cases. (a). Case l: coxofemoral joint MRI (frontal projection) showed left coxofemoral intra-articular effusion (continuous arrows) with oedematous-flogistic muscular involvement (dotted arrow) but no bone involvement. (b). Case II: Coxofemoral joint MRI (axial projection) showed mild right hip joint effusion (continuous arrows) and fasciitis muscular effusion (dotted arrows); no bone lesions were detected 
isolated by synovial fluid culture [17].To our knowledge, less than 50 cases are described in the literature, combining adult and pediatric patients $[1-7,17,18]$. PMA is defined as the presence of septic arthritis without meningeal signs or meningococcaemia and the detection of $N$. meningitidis in synovial fluid and/or blood analysis. The knee is described as the most affected joint followed by the ankle. Approximately $50 \%$ of patients were children less than 4 years of age [18]. Despite its unusual presentation it is important not to miss a diagnosis of meningococcal septic arthritis as appropriate treatment and prophylaxis for contacts are required.

When facing uncommon or no specific presentations (e.g., pneumonia, septic arthritis), clinicians are less likely to suspect meningococcal disease and thus to submit clinical specimens for laboratory confirmation than in classic IMD cases (meningitis or sepsis). Moreover, in the context of an unusual presentation is more likely to collect specimens for microbiological identification after having started empirical antimicrobial treatment, reducing the likelihood for the pathogen isolation. This suggests that a proportion of laboratory diagnosis of PMA might be lost and that disease burden PMA might be underestimated. RT-PCR improves the diagnostic rates after having started antibiotic treatment and also allows the characterization of the Nm serogroups, which contributes to a better understanding of the meningococcal disease epidemiology.

A rise in the number of NmY infections has been observed in the last 20 years in several countries [19, 20]. In Italy, despite an overall invariable incidence of IMD of 0.3 cases/100,000 inhabitants since 2007, an increased proportion of NmY has been observed [21]. Similarly, to other European countries, serogroup $B$ and $C$ are responsible for the majority of cases, although the proportion of NmY IMD cases has steadily increased over the years [20], surging from $2 \%$ in 2007 to $17 \%$ in 2013, in particular among 5- to 14-year-old patients, which is reported to be the most affected group since 2008 [21].

In our laboratory, we routinely use RT-PCR tests on blood and other normally sterile fluids - as previously described [9] - in association with culture to enhance the reliability of the IMD laboratory diagnosis. In the cases we presented, even though arthrocentesis was performed after having started antimicrobial therapy, RT-PCR on synovial fluid was able to detect NmY ST23/ clusterA3, whereas the cultures resulted negative.

According to the pubMLST database, among invasive NmY the ST-23/cluster A3 complex (cc23) is the major cluster complex (cc) reported in Italy. The ST-23/cluster A3 complex could account for more than $85 \%$ of Italian IMD cases caused by NmY (http://pubmlst.org/neisseria) and five of the total of six cases of meningococcal arthritis caused by NmY are reported (from 2011 to 2018) in
Europe were characterized as ST-23/cluster A3 complex (cc23). A limitation of this analysis is that the submission of isolates to the database is voluntary, which might represent a selection bias and underrepresent the real incidence rate.

In conclusion, our report aims to raise awareness among physicians of the atypical presentations of IMD and highlights the diagnostic sensitivity improvements added by the routine use of RT-PCR for IMD, also in the event of unusual presentations, such as PMA. Better quality in laboratory confirmation of IMD is necessary to improve the understanding of the epidemiological features of $\mathrm{Nm}$ disease. Currently $\mathrm{NmY}$ is not part of the Italian Immunization Program for children under 11-years-old and, based on our limited data we cannot draw conclusions about a dedicated national vaccination strategy. However, we believe that a more accurate surveillance based on cultural and molecular methods would better frame the diffusion of the different meningococcal serogroups allowing a review of the current vaccination strategies. A better estimation of IMD rates, based on active and appropriate surveillance, would likely help in the development of an effective national vaccination program, which remains the best control strategy to prevent invasive meningococcal disease.

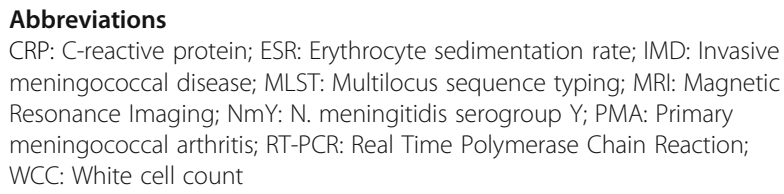

\section{Acknowledgements}

Data available via the Neisseria Multi Locus Sequence Typing website (http:// pubmlst.org/neisseria) developed by Keith Jolley and sited at the University of Oxford (Jolley KA, Maiden M. BIGSdb: scalable analysis of bacterial genome variation at the population level. BMC Bioinformatics 2010; 11: 595).

\section{Funding}

This research did not receive any specific grant from funding agencies in the public, commercial, or not-for-profit sectors.

\section{Availability of data and materials}

The data is available upon request and corresponding author should be contacted if someone wants to request the data.

\section{Authors' contributions}

AM, DS evaluated the patients during their hospitalization. SR carried out the immunological evaluation. SR, AM, DS and GI collected the clinical and immunological data, revised literature, wrote the first draft of the article, and critically revised it. MM and FN carried out the molecular diagnosis. CA, as expert, critically revised the article and gave the final approval for submission. All authors read and approved the final manuscript.

\section{Ethics approval and consent to participate}

The study was approved by the local review board. A specific approval by the local ethical committee was not required because all analyses included in this study had been performed as part of the routine clinical activity. All results have been anonymized. 


\section{Consent for publication}

Written informed consent was obtained from the guardians of the patients for the publication of this case report and any related images.

\section{Competing interests}

All authors declare that there are no competing interest.

\section{Publisher's Note}

Springer Nature remains neutral with regard to jurisdictional claims in published maps and institutional affiliations.

\section{Author details}

${ }^{1}$ Immunology Division, Section of Pediatrics, Department of Health Sciences, University of Florence and Meyer Children's University Hospital, Florence, Italy. ${ }^{2}$ Section of Paediatrics, Meyer Children's University Hospital, Florence, Italy. ${ }^{3}$ Section of Pediatrics, Department of Neurofarba, University of Florence and Meyer Children's University Hospital, Florence, Italy.

Received: 19 March 2018 Accepted: 10 December 2018

Published online: 27 December 2018

\section{References}

1. Barahona M, Catalan J, Sato Y, Hinzpeter J. Primary meningococcal type C arthritis: a case report and literature review. Case Rep Orthop. 2017;2017: 4696014. https://doi.org/10.1155/2017/4696014.

2. O'Sullivan D, Linnane B, Mostyn A, Jonathan N, Lenihan M, O'Connell NH, Dunne CP. Detection of Neisseria meningitidis in a paediatric patient with septic arthritis using multiplexed diagnostic PCR targeting meningitis/ encephalitis (ME). Ann Clin Microbiol Antimicrob. 2018;17(1):14. https://doi. org/10.1186/s12941-018-0268-7.

3. Straticiuc S, Ignat A, Hanganu E, Lupu W, Ciubara AB, Cretu R. Neisseria meningitidis Serogroup C causing primary arthritis in a child: case report. Medicine (Baltimore). 2016;95(5):e2745.

4. Sordelli N, Orlando N, Neyro S, Echave C, Procopio A, Fallo A, López EL Primary meningococcal arthritis in pediatrics. Report of nine cases Arch Argent Pediatr. 2011;109(2):150-4. https://doi.org/10.1590/S032500752011000200010.

5. De Laere E, Berghs B, Gordts B, Van Landuyt H. Primary meningococcal arthritis of the hip in an immunocompetent adolescent. Acta Clin Belg. 2002;57(6):345-8.

6. Giamarellos-Bourboulis EJ, Grecka P, Petrikkos GL, Toskas A, Katsilambros N Primary meningococcal arthritis: case report and review. Clin Exp Rheumatol. 2002:20(4):553-4

7. Cartolano GL, Le Lostec Z, Chéron M, Boisivon A, Welker $Y$, Mornet P. Primary Neisseria meningitidis arthritis of the knee without meningitis: contribution of synovial fluid culture in blood-culture vial. Rev Med Interne. 2001:22(1):75-8.

8. Faust SN, Clark J, Pallett A, Clarke NM. Managing bone and joint infection in children. Arch Dis Child. 2012;97(6):545-53.

9. Azzari C, Nieddu F, Moriondo M, Indolfi G, Canessa C, Ricci S, et al. Underestimation of invasive meningococcal disease in Italy. Emerg Infect Dis. 2016;22(3):469-75.

10. Bosis S, Mayer A, Esposito S. Meningococcal disease in childhood: epidemiology, clinical features and prevention. J Prev Med Hyg. 2015;56(3): E121-4

11. Dunne EM, Mantanitobua S, Singh SP, Reyburn R, Tuivaga E, Rafai E, Tikoduadua L, Porter B, Satzke C, Strachan JE, Fox KK, Jenkins KM, Jenney A, Baro S, Mulholland EK, Kama M, Russell FM. Real-time qPCR improves meningitis pathogen detection in invasive bacterial-vaccine preventable disease surveillance in Fiji. Sci Rep. 2016;6:39784.

12. Heinsbroek E, Ladhani S, Gray S, Guiver M, Kaczmarski E, Borrow R, Ramsay $M$. Added value of PCR-testing for confirmation of invasive meningococcal disease in England. J Inf Secur. 2013;67(5):385-90. https://doi.org/10.1016/j. jinf.2013.06.007.

13. Edge C, Waight P, Ribeiro S, Borrow R, Ramsay M, Ladhani S. Clinical diagnoses and outcomes of 4619 hospitalised cases of laboratory-confirmed invasive meningococcal disease in England: linkage analysis of multiple national databases. J Inf Secur. 2016;73(5):427-36.
14. Papavasileiou K, Papavasileiou E, Tzanakaki G, Voyatzi A, Kremastinou J, et al. Acute bacterial meningitis cases diagnosed by culture and PCR in a children's hospital throughout a 9-year period (2000-2008) in Athens, Greece. Mol Diagn Ther. 2011:15(2):109-13.

15. Centers for Disease Control and Prevention. Laboratory Methods for the Diagnosis of Meningitis Caused by Neisseria meningitidis, Streptococcus pneumoniae, and Haemophilus influenzae. Second edition (2011). Chapter 10: PCR for Detection and Characterization of Bacterial Meningitis Pathogens: Neisseria meningitidis, Haemophilus influenzae, and Streptococcus pneumoniae.

16. Young TP, Maas L, Thorp AW, Brown L. Etiology of septic arthritis in children: an update for the new millenium. Am J Emerg Med. 2011;29:899-902.

17. Bilavsky E, Yarden-Bilavsky H, Zevit N, Amir J. Primary meningococcal arthritis in a child: case report and literature review. Scand J Infect Dis. 2006 38(5):396-9.

18. Weels M, Gibbons RB. Primary meningococcal arthritis. Case report and review of the literature. Mil Med. 1997:162:769-72.

19. Bröker M, Emonet S, Fazio C, Jacobsson S, Koliou M, Kuusi M, et al. Meningococcal serogroup $Y$ disease in Europe: continuation of high importance in some European regions in 2013. Hum Vaccin Immunother. 2015;11(9):2281-6.

20. Säll O, Stenmark B, Glimåker M, Jacobsson S, Mölling P, Olcén P, et al. Clinical presentation of invasive disease caused by Neisseria meningitidis serogroup $Y$ in Sweden, 1995 to 2012. Epidemiol Infect. 2017:145(10):2137-43.

21. Fazio C, Neri A, Renna G, Vacca P, Antonetti R, Barbui A, et al. Persistent occurrence of serogroup Y/sequence type (ST)-23 complex invasive meningococcal disease among patients aged five to 14 years, Italy, 2007 to 2013. Euro Surveill. 2015:20(45).

Ready to submit your research? Choose BMC and benefit from:

- fast, convenient online submission

- thorough peer review by experienced researchers in your field

- rapid publication on acceptance

- support for research data, including large and complex data types

- gold Open Access which fosters wider collaboration and increased citations

- maximum visibility for your research: over $100 \mathrm{M}$ website views per year

At $\mathrm{BMC}$, research is always in progress.

Learn more biomedcentral.com/submissions 